\title{
Características clínicas y demográficas de los pacientes incidentes en diálisis crónica y su relación con el ingreso programado a diálisis
}

\author{
Clinical and demographic characteristics of \\ incident patients on chronic dialysis and its \\ relationship to the planned admission to dialysis
}

\author{
Carlos Yesid Coronado, Jadith Cristina lombo, Indira Correa, \\ Natalia QuinTERo・IBAgué (Colombia)
}

\section{Resumen}

Objetivo: describir las características clínicas y demográficas de los pacientes incidentes en diálisis, y su relación con el inicio programado de diálisis en la Unidad Renal del Tolima en la ciudad de Ibagué.

Contexto y tipo de estudio: estudio observacional de corte transversal. Población de referencia conformada por los pacientes de nefrología atendidos en 10 hospitales de la ciudad de Ibagué y por los pacientes que asisten a consulta externa de nefrología de la Unidad Renal del Tolima.

Material y métodos: información recolectada por los investigadores mediante revisión de historias clínicas, variables sociodemográficas y clínicas que determinan las características del ingreso a diálisis.

Resultados: se analizaron 74 pacientes con mediana de edad 65 años. En 82.4\% la modalidad dialítica inicial fue hemodiálisis. El $93.5 \%$ de los pacientes incidentes a hemodiálisis inició en forma no programada. El $43.2 \%$ del total de incidentes a diálisis tuvo valoración previa por nefrología, y de éstos el $71.9 \%$ ingresó de forma no programada a diálisis. El 50\% de los pacientes que vive fuera de Ibagué y el $43.7 \%$ de los que lo hace en zona rural seleccionan hemodiálisis como terapia definitiva.

Se asocian en forma estadísticamente significativa al inicio programado de diálisis: cuidado previo por nefrología, régimen de salud contributivo, nefropatía diabética, condición ambulatoria al inicio de diálisis, y diálisis peritoneal como modalidad dialítica inicial.

Conclusiones: los pacientes incidentes tuvieron una edad avanzada, y en su mayoría ingresaron estando hospitalizados y sin acceso dialítico definitivo inclusive dentro del grupo de pacientes con manejo previo por nefrología. (Acta Med Colomb 2013; 38: 138-142).

Palabras clave: insuficiencia renal crónica, diálisis, epidemiología.

\footnotetext{
Abstract

Objective: to describe the clinical and demographic characteristics of incident patients in dialysis, and its relation to the programmed start of dialysis in the Renal Unit of Tolima in Ibagué.

Context and type of study: cross-sectional observational study. Reference population comprised nephrology patients treated at 10 hospitals in the city of Ibague and patients attending the outpatient nephrology Renal Unit of Tolima.

Material and methods: information collected by researchers by reviewing medical records, demographic and clinical variables that determine the characteristics of admission to dialysis.

Results: we analyzed 74 patients with a mean age of 65 years. In $82.4 \%$ the initial dialysis modality was hemodialysis. $93.5 \%$ of patients starting hemodialysis started in a nonprogrammed way. $43.2 \%$ of all incident patients to dialysis had prior nephrology assessment, and of these, $71.9 \%$ were admitted for dialysis in a nonprogrammed way. $50 \%$ of patients who live outside of Ibague and $43.7 \%$ of those in rural areas select hemodialysis as a definitive therapy.

Factors associated in a statistically significant way to the programmed start of dialysis, are: prior nephrology care, contributory health scheme, diabetic nephropathy, ambulatory status at the start of dialysis, and peritoneal dialysis as initial dialysis modality.
}

Dr. Carlos Yesid Coronado Suescún: Especialista en Medicina Interna, Nefrología y Epidemiología; Dra. Jadith Cristina Lombo Caicedo: Enfermera, Candidata a Magiste en Enfermería; Dra. Indira Correa: Médica; Dra. Natalia Quintero Tobón: Enfermera, Especialista en Epidemiología. Unidad Renal del Tolima. Ibagué (Colombia). Correspondencia. Dr. Carlos Yesid Coronado Suescún.

E-mail: cycoronado@yahoo.com Recibido: 17/VI/2012 Aceptado: 27/VI/2013 
Conclusions: incident patients had an advanced age, and were mostly admitted being hospitalized without definitive dialysis access even within the group of patients with prior management by nephrology. (Acta Med Colomb 2013; 38: 138-142).

Keywords: Chronic renal failure, dialysis, epidemiology.

\section{Introducción}

Para el año 2005 Colombia tenía una prevalencia estimada de diálisis de 355 pacientes por millón de habitantes (1), y para el año 2010 el Fondo Colombiano de Enfermedades de Alto Costo informa 19549 pacientes en diálisis que corresponde a una prevalencia estimada de 434 pacientes en diálisis por millón de habitantes (2). Esto refleja un incremento en la prevalencia en diálisis de $22.3 \%$ en cinco años. Estos datos son consistentes con los reportados por los registros UKRR (United Kingdom Renal Registry), USRDS (United States Renal Data System), EDTA/ERA (European Dialysis and Transplant Association/European Renal Association), y ANZDATA (Australian and New Zeland renal database) que informan una incidencia mediana de ingreso de pacientes a diálisis crónica de 136 pacientes por millón de habitantes con un incremento anual de 3.5\%, y una prevalencia en el rango entre 99 pacientes por millón de habitantes en Bangladesh y 2288 pacientes por millón de habitantes en Taiwan, con una mediana de incremento anual en la prevalencia de $3.9 \%$ (3).

Estos datos junto con el alto costo del tratamiento dialítico hacen de la insuficiencia renal crónica la enfermedad con mayor impacto financiero y epidemiológico en el sistema general de seguridad social en salud de Colombia (corresponde a $64 \%$ del costo total por enfermedades catastróficas) (4).

El ingreso programado a diálisis es importante porque se asocia con menor morbilidad y mortalidad, y reducción de costos en diálisis (5). En Colombia, Alarcón et al. (6) describieron las características clínicas y paraclínicas de los pacientes incidentes en diálisis de dos unidades renales de la ciudad de Medellín y encontraron que $51.4 \%$ de los pacientes ingresó de forma urgente, y que solamente $2.4 \%$ de los pacientes incidentes en hemodiálisis lo hizo mediante un acceso dialítico definitivo.

Además de las características clínicas, queremos describir las características demográficas y de aseguramiento en salud, así como su relación con el inicio programado de diálisis en un centro único de diálisis en la ciudad de Ibagué. Esto deberá permitirnos desarrollar estrategias de manejo del paciente prediálisis para mejorar las condiciones de salud de la población que inicia tratamiento dialítico crónico.

\section{Material y métodos}

Es un estudio observacional de corte transversal en el que además de describir las características clínicas y sociodemográficas de los pacientes que ingresan a diálisis, se estudia la relación que éstas tienen con el ingreso programado a diálisis.

El estudio se llevó a cabo con los pacientes que ingresaron a diálisis en un centro único no hospitalario de la ciudad de Ibagué donde se realizan hemodiálisis, diálisis peritoneal y consulta externa de nefrología, y que tiene convenio para valorar los pacientes que requieren manejo por nefrología provenientes de 10 centros hospitalarios de diferente nivel de complejidad en la ciudad de Ibagué.

La población de referencia estuvo constituida por aquellos pacientes atendidos en todos los hospitales de diferente nivel de complejidad de la ciudad de Ibagué, y por la población que asiste a consulta externa en nuestro centro de diálisis. La población de estudio fue conformada por aquellos pacientes que iniciaron diálisis en la modalidad de hemodiálisis o diálisis peritoneal en el centro de diálisis durante el año 2011. No se calculó tamaño muestral debido a que se incluyeron todos los pacientes que cumplieron con los criterios de selección establecidos.

La información fue recolectada por los investigadores mediante la revisión de las historias clínicas aplicando un formulario diseñado para este fin.

\section{Variables sociodemográficas}

Edad al inicio del tratamiento dialítico, sexo, ciudad de origen que corresponde al lugar de residencia del paciente al momento de iniciar diálisis y que se clasificó como Ibagué o una ciudad diferente a Ibagué; el dato de vivienda rural o urbana, se tomó de la información consignada por trabajo social; el régimen de salud al que pertenece el paciente fue clasificado como contributivo o subsidiado.

\section{Variables clínicas}

Etiología de la enfermedad renal crónica, cuidado previo por nefrología clasificado en tres categorías según si el paciente había sido valorado por nefrología en los tres meses previos a su ingreso a diálisis, más allá de estos tres meses, o si nunca había sido valorado por nefrología; albúmina, paratohormona intacta (PTHi), y tamización para hepatitis B, C y VIH tomados dentro de los primeros 30 días de haber ingresado a diálisis. La modalidad dialítica inicial corresponde al primer tipo de diálisis con que es tratado el paciente. En el centro de diálisis, tanto los pacientes que ingresan de forma programada como los que lo hacen de forma no programada, reciben educación en las dos modalidades de diálisis. La modalidad de diálisis elegida una vez recibida esta educación la llamamos terapia dialítica 
elegida. No realizamos seguimiento para verificar si esta terapia dialítica elegida correspondió a la terapia real en la que ya queda el paciente.

Se definió ingreso programado como el ingreso del paciente que tiene accesos dialíticos definitivos al momento de la primera hemodiálisis: fístula arteriovenosa (FAV) o prótesis vascular. En el caso de la diálisis peritoneal se consideró ingreso programado si el catéter peritoneal se implantó al menos tres semanas antes del inicio de la diálisis peritoneal contando desde el momento en que se implanta el catéter hasta cuando se formula la diálisis con el objetivo de cumplir con la meta de aclaramiento de solutos (Kt/V). Este criterio para diálisis peritoneal lo definimos pensando en el tiempo adecuado para que los pacientes o cuidadores reciban entrenamiento en diálisis peritoneal.

En las historias clínicas no estaba registrada de forma sistemática la hemoglobina inicial, ni la creatinina previa a la realización de la primera diálisis por lo que estas variables no se incluyeron en este estudio.

\section{Criterios de inclusión}

Pacientes mayores de 14 años de ambos sexos.

Pacientes con enfermedad renal crónica que iniciaron terapia dialítica crónica, tanto hemodiálisis como diálisis peritoneal entre el $1^{\circ}$ de enero y el 31 de diciembre de 2011.

\section{Criterios de exclusión}

Pacientes que iniciaron diálisis en otra institución y posteriormente fueron transferidos al centro de diálisis para continuar tratamiento dialítico.

\section{Análisis estadístico}

Se hizo análisis descriptivo a la totalidad de las variables incluidas en la base de datos. Las variables discretas fueron expresadas en frecuencias absolutas y porcentajes, las continuas como promedios y desviaciones estándar o mediana y rango intercuartil. Las diferencias en las características de los pacientes se estudiaron con la prueba Chi-cuadrado para las variables discretas; y prueba-t para variables continuas de muestras independientes, si la distribución fue normal. Cuando la distribución no fue normal las diferencias se estudiaron con la prueba Mann-Whitney. El nivel de significancia fue $\mathrm{p}<0,05$. Todos los análisis se realizaron con el paquete estadístico Stata-11. Las gráficas se realizaron con Excel.

\section{Resultados}

Setenta y cuatro pacientes cumplieron con los criterios de participación en el estudio. La media de la edad fue 61.8 años, y 50\% de los pacientes fue mayor de 65 años. El 74.3\% perteneció al sexo masculino y $25.7 \%$ al femenino. La edad mediana en hombres fue 66 años y en mujeres 63 años.

La etiología de la nefropatía fue: diabetes (37.8\%), hipertensión arterial (25.7\%), glomerulonefritis (14.9\%), desconocida (10.8\%), obstructiva (8.1\%), y enfermedad poliquística $(2.7 \%)$.

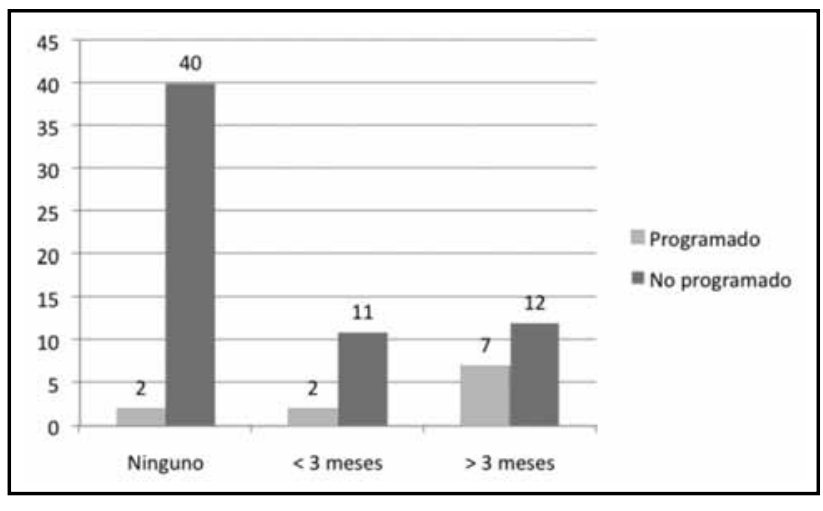

Figura 1. Distribución de frecuencias absolutas según el cuidado previo por nefrología y el tipo de ingreso

La Tabla 1 resume las características clínicas y demográficas de los pacientes.

La Tabla 1 muestra que existe asociación significativa entre el tipo de ingreso a diálisis y el cuidado previo por nefrología, el régimen de salud, la nefropatía diabética como etiología, la condición inicial, y la modalidad dialítica inicial.

En la Figura 1 se observa la distribución del ingreso programado o no programado frente a la ausencia de cuidado previo por nefrología y al cuidado previo por nefrología tanto menor de tres meses como mayor de tres meses.

De los 32 pacientes que tuvieron cuidado previo por nefrología, nueve ingresaron de forma programada (28.1\%). Dos pacientes no tuvieron remisión a nefrología pero tuvieron ingreso programado a diálisis. Esto se explica porque son pacientes con indicación dialítica desde la primera valoración de nefrología en quienes se implantó el catéter peritoneal y pudieron esperar tres semanas para iniciar diálisis peritoneal crónica ambulatoria.

De todos los pacientes, la terapia dialítica elegida fue hemodiálisis en $50 \%$ de los casos, y diálisis peritoneal en el otro $50 \%$. Esta distribución fue exactamente igual, cuando el análisis se realizó según si el paciente vive en la ciudad de Ibagué o fuera de Ibagué.

De los 16 pacientes que viven en zona rural, siete (43.7\%) eligieron hemodiálisis como la modalidad dialítica definitiva.

La media de albúmina fue $3.2 \pm 0.66 \mathrm{~g} \%$ y $62 \%$ de los paciente tuvo albúmina $<3.5 \mathrm{~g} / \mathrm{dL}$.

La mediana de PTHi fue $210 \mathrm{pg} / \mathrm{mL}$ con rango intercuartil de $259.3 \mathrm{pg} / \mathrm{mL}$, y $39 \%$ de los pacientes tuvo más de $300 \mathrm{pg} / \mathrm{mL}$.

Ningún paciente fue positivo en la tamización para VIH, hepatitis B o hepatitis C.

\section{Discusión}

Llama la atención que la mediana de edad de los pacientes incidentes en diálisis fue 65 años. Es alta si tenemos en cuenta que el estudio DOC realizado en Colombia con pacientes incidentes entre los años 2001 y 2003 informó 
Tabla 1. Características de los pacientes según tipo de ingreso a diálisis.

\begin{tabular}{|c|c|c|c|c|}
\hline Variable & $\begin{array}{l}\text { Total de pacientes } \\
\qquad(n=74)\end{array}$ & $\begin{array}{c}\text { Ingreso programado } \\
(\mathbf{n = 1 1})\end{array}$ & $\begin{array}{l}\text { Ingreso no programado } \\
\qquad(\mathrm{n}=63)\end{array}$ & $\mathbf{P}$ \\
\hline $\begin{array}{l}\text { Sexo } \\
\text { Masculino } \\
\text { Femenino }\end{array}$ & $\begin{array}{l}55(74.3 \%) \\
19(25.7 \%)\end{array}$ & $\begin{array}{l}8(72.7 \%) \\
3(27.3 \%)\end{array}$ & $\begin{array}{l}47(74.6 \%) \\
16(25.4 \%)\end{array}$ & 0.896 \\
\hline Edad (RI) años & $64.5(15)$ & $64(14)$ & $65(19)$ & 0.903 \\
\hline $\begin{array}{l}\text { Ciudad de origen } \\
\text { Ibagué } \\
\text { Otra ciudad }\end{array}$ & $\begin{array}{l}36(48.6 \%) \\
38(51.4 \%)\end{array}$ & $\begin{array}{l}5(54.5 \%) \\
6(45.5 \%)\end{array}$ & $\begin{array}{l}31(49.2 \%) \\
32(50.8 \%)\end{array}$ & 0.819 \\
\hline $\begin{array}{l}\text { Vivienda } \\
\text { Urbana } \\
\text { Rural }\end{array}$ & $\begin{array}{l}58(78.4 \%) \\
16(21.6 \%)\end{array}$ & $\begin{array}{l}9(81.8 \%) \\
2(18.2 \%)\end{array}$ & $\begin{array}{l}49(77.8 \%) \\
14(22.2 \%)\end{array}$ & 0.765 \\
\hline $\begin{array}{l}\text { Cuidado previo por nefrología } \\
\text { Si } \\
\text { No }\end{array}$ & $\begin{array}{l}32(43.2 \%) \\
42(56.8 \%)\end{array}$ & $\begin{array}{l}9(81.8 \%) \\
2(18.2 \%)\end{array}$ & $\begin{array}{l}23(36.5 \%) \\
40(63.5 \%)\end{array}$ & 0.005 \\
\hline $\begin{array}{l}\text { Régimen de salud } \\
\text { Subsidiado } \\
\text { Contributivo }\end{array}$ & $\begin{array}{l}46(62.2 \%) \\
28(37.8 \%)\end{array}$ & $\begin{array}{l}3(27.3 \%) \\
8(72.7 \%)\end{array}$ & $\begin{array}{l}43(68.2 \%) \\
20(31.8 \%)\end{array}$ & 0.010 \\
\hline $\begin{array}{l}\text { Nefropatía diabética } \\
\text { Si } \\
\text { No }\end{array}$ & $\begin{array}{l}28(37.8 \%) \\
46(62.2 \%)\end{array}$ & $\begin{array}{l}9(81.8 \%) \\
2(18.2 \%)\end{array}$ & $\begin{array}{l}19(30.2 \%) \\
44(69.8 \%)\end{array}$ & 0.001 \\
\hline $\begin{array}{c}\text { Condición inicial } \\
\text { Hospitalizado } \\
\text { Ambulatorio }\end{array}$ & $\begin{array}{l}61(82.4 \%) \\
13(17.6 \%)\end{array}$ & $\begin{array}{l}3(27.3 \%) \\
8(72.7 \%)\end{array}$ & $\begin{array}{c}58(92.1 \%) \\
5(7.9 \%)\end{array}$ & 0.000 \\
\hline PTHi (RI) pg/mL & $210(259.3)$ & $186.9(216.5)$ & $231.6(262.7)$ & 0.621 \\
\hline Albúmina (DS) g/dL & $3.20(0.66)$ & $3.33(0.77)$ & $3.18(0.65)$ & 0.747 \\
\hline $\begin{array}{l}\text { Modalidad dialítica inicial } \\
\text { Diálisis peritoneal } \\
\text { Hemodiálisis }\end{array}$ & $\begin{array}{l}13(17.6 \%) \\
61(82.4 \%)\end{array}$ & $\begin{array}{l}7(63.6 \%) \\
4(36.4 \%)\end{array}$ & $\begin{array}{c}6(9.5 \%) \\
57(90.5 \%)\end{array}$ & 0.000 \\
\hline \multicolumn{5}{|c|}{$\begin{array}{l}\text { P, es el valor estadístico P referente a la diferencia entre ingreso programado y no programado en cada una de las variables estudiadas } \\
\text { RI (rango intercuartil), es precedido por la mediana } \\
\text { DS (desviación estándar), es precedida por la media }\end{array}$} \\
\hline
\end{tabular}

una mediana de 57 años para hemodiálisis y 54 años para diálisis peritoneal (1). Además la mediana de 65 años es tan alta como en los países europeos que tienen una distribución poblacional menos joven que la nuestra. Italia es el país con la mediana de edad más alta: 67 años (3).

En el presente trabajo $93.5 \%$ de los pacientes incidentes a hemodiálisis lo hicieron de forma no programada, resultado similar al encontrado por Alarcon et al. en Medellín $(97.1 \%)$ (6) y superior a $48.6 \%$ reportado en un estudio español (7). La diferencia con este estudio podría deberse a factores modificables como la remisión oportuna a nefrología, la disponibilidad de cirujanos para la confección de los accesos vasculares, y la mejor adherencia del paciente a las recomendaciones del nefrólogo. También existen factores no modificables causales del ingreso no programado a diálisis como pueden ser el curso no lineal en la progresión de la enfermedad renal recientemente descrito por $\mathrm{Li} \mathrm{L}$. et al. (8), o la presencia de eventos intercurrentes no previsibles.

En este trabajo $71.9 \%$ de los 32 pacientes que recibieron cuidado previo por nefrología tuvo ingreso no programado a diálisis. Esto puede ser en parte a una remisión tardía a nefrología debido a que 13 pacientes fueron remitidos con menos de tres meses de anticipación a su ingreso a diálisis. Existen series que muestran mejores resultados. Gomis et al en España, informan que 19\% de los pacientes con cuidado predialítico ingresa de forma no programada (9). Inaguma et al. en un estudio no aleatorizado realizado en Japón, encontraron 4\% de inicio no programado de diálisis en pacientes que recibieron un programa estructurado y multidisciplinario de educación frente a $25 \%$ en un grupo de pacientes con manejo predialítico convencional (10). Aunque por su diseño, el estudio de Inaguma et al. no permite sacar conclusiones sobre el impacto del cuidado previo por nefrología en el ingreso programado a diálisis, los resultados globales de dicha cohorte son similares a los resultados del estudio español previamente citado. Los pacientes incidentes del presente estudio recibieron cuidado previo por nefrología en diferentes instituciones y no disponemos de datos para analizarlo.

El $82.2 \%$ de los pacientes que ingresa a diálisis lo hace estando hospitalizados. Esto tiene un impacto importante en costos y refleja el carácter de urgencia de esta situación clínica, en que ingresan los pacientes a diálisis, además de que podría ser un factor adverso en la supervivencia del paciente. 
Es llamativo que $50 \%$ de los pacientes que viven fuera de Ibagué y $43.7 \%$ de los pacientes que lo hace en zona rural, seleccionen hemodiálisis como terapia definitiva. La necesidad de desplazarse grandes distancias tres veces por semana debería motivar la selección de diálisis peritoneal en este grupo de pacientes.

Como se dijo antes, en este trabajo se encontró asociación significativa entre el inicio programado de diálisis con: cuidado previo de nefrología, régimen de salud, nefropatía diabética, condición inicial (hospitalizado o ambulatorio) y modalidad dialítica inicial.

Arora et al. (11) reportaron que existe menor riesgo de remisión tardía a nefrología entre los beneficiarios de Medicare en Estados Unidos que entre aquellos que no tienen este seguro médico. Nuestro estudio halló una asociación entre el régimen de salud subsidiado y menor frecuencia de inicio programado de diálisis. El estado socioeconómico, no evaluado en este trabajo, puede ser un factor de confusión que sea responsable de esta asociación. Esto debe evaluarse en futuros estudios.

La asociación entre nefropatía diabética e inicio programado de diálisis posiblemente se deba a que la tamización para enfermedad renal que se realiza en los pacientes diabéticos permite una identificación más temprana de la enfermedad renal.

La asociación encontrada en este trabajo entre la selección de diálisis peritoneal como terapia de inicio y el ingreso programado a diálisis da soporte en nuestro medio a la estrategia propuesta por otros autores de priorizar la referencia temprana a nefrología para incrementar la utilización de esta modalidad dialítica (12). Se ha reportado un aumento de la elección de diálisis peritoneal cuando los pacientes reciben educación sobre las modalidades de diálisis alcanzando $31 \%$ según Marrón et al. (13), y 50\% según Prichard et al. (14).

Una limitante del presente estudio es el pequeño número de pacientes, y el carácter retrospectivo del mismo. Además, que sus resultados no son generalizables por tratarse de un único centro de diálisis. Un estudio multicéntrico con un mayor número de pacientes posiblemente permitiría generalizar los resultados.

\section{Conclusiones}

Los pacientes incidentes a diálisis en el presente trabajo tienen una edad avanzada, y en su mayoría ingresan por hemodiálisis, de forma no programada y estando hospitalizados. En el grupo de pacientes con cuidado previo por nefrología es predominante el ingreso no programado a diálisis. Un importante porcentaje de pacientes que vive en zona rural o lejos del centro de diálisis selecciona hemodiálisis como modalidad dialítica definitiva.

Se asocian significativamente al inicio programado de diálisis, los cuatro factores que ya hemos visto antes, es decir, cuidado previo por nefrología, régimen de salud contributivo, nefropatía diabética, condición ambulatoria al inicio de diálisis y diálisis peritoneal como modalidad dialítica inicial.

\section{Referencias}

1. Sanabria M, Muñoz J, Trillos C, Hernández G, Latorre C, Díaz CS, et al Dialysis outcomes in Colombia (DOC) study: A comparison of patient survival on peritoneal dialysis vs hemodialysis in Colombia. Kidney Internat 2008; 73: S165-S72.

2. Ministerio de Hacienda y Crédito Público. Cuenta de Alto Costo. República de Colombia. Ministerio de Salud y Protección Social.. Epidemiología y estadísticas ERC. Informe de terapia dialítica en Colombia 2010 en http://www. cuentadealtocosto.org/.

3. Donovan K, Ford D, Schalkwyk D, Ansell D. UK Renal Registry 12th Annual Report (2009): Chapter 16 International Comparasions with the UK RRT Programme. Nephron Clin Pract 2010; 115(suppl 1): c309-c20.

4. Chicaiza L. Fallas del mercado de la salud en Colombia: el caso de la insuficiencia renal crónica. Revista de Economía Institucional 2005; 7(12): 191-208.

5. Sesso R, Belasco AG. Late diagnosis of chronic renal failure and mortality on maintenance dialysis. Nephrol Dial Transplant 1996; (11): 2417-20.

6. Alarcón JC, Lopera JM, Montejo JD, Henao CM, Rendón G. Perfil epidemiológico de pacientes en diálisis, CTRB y RTS sucursal Medellín 2000-2004. Acta Med Colomb 2006; 31: 4-12.

7. Marrón B, Ortiz A, Sequera P, Martín-Reyes G, Arriba G, Lamas JM, et al. Impact of end-stage renal disease care in planned dialysis start and type of enal replacement therapy-a Spanish multicentre experience. Nephrol Dial Transplant 2006; 21(Suppl 2): ii51-ii5.

8. Li L, Astor BC, Lewis J, Hu B, Appel L, Lipkowitz MS, et al. Longitudinal progression trajectory of GFR among patients with CKD. Am J Kindey Dis 2012; 59(4): $504-12$

9. Gomis A, Teruel J, Fernández M, Rivera M, Rodríguez N, Jiménez A, et al. Causas de inicio no programado del tratamiento renal sustitutivo con hemodiálisis. Nefrología 2011; 31(6): 733-7.

10. Inaguma D TM, Shinjo I, Suzuki S, Mishima T, Inaba S. Effect of an educational program on the predialysis period for patients with chronic renal failure. Clin Exp Nephrol 2006; 10: 274-8.

11. Arora P, Obrador GT, Ruthazer R, Kausz AT, Meyer KB, Jenuleson CS, et al. Prevalence, predictors, and consequences of late nephrology referral at a tertiary care center. J Am Soc Nephrol 1999; (10): 1281-6.

12. Heaf J. Underutilization of Peritoneal Dialysis. JAMA 2004; 291(6): 740-2.

13. Marrón B, Martínez-Ocaña JC, Salgueira M, Barril G, Lamas JM, Martín $\mathbf{M}$, et al. Analysis of patient flow into dialysis: role of education in choice of dialysis modality. Perit Dial Internat 2005; 25: S56-S9.

14. Prichard SS. Treatment modality selection in 150 consecutive patients starting ESRD therapy. Perit Dial Internat 1996; 16: 69-72. 